\title{
Down-regulation of uPAR and Cathepsin B retards cofilin dephosphorylation
}

\author{
CHRISTOPHER S. GONDI ${ }^{1}$, NEELIMA KANDHUKURI ${ }^{1}$, SHAKUNTALA KONDRAGANTI ${ }^{1}$, \\ MEENA GUJRATI ${ }^{2}$, WILLIAM C. OLIVERO ${ }^{3}$, DZUNG H. DINH ${ }^{3}$ and JASTI S. RAO ${ }^{1,3}$ \\ ${ }^{1}$ Program of Cancer Biology, Department of Biomedical and Therapeutic Sciences, ${ }^{2}$ Department of Pathology, \\ ${ }^{3}$ Department of Neurosurgery, University of Illinois College of Medicine at Peoria, Peoria, IL, USA
}

Received September 2, 2005; Accepted October 24, 2005

\begin{abstract}
Cathepsin B and UPAR play key roles in cancer cell migration and invasion. Here, we demonstrate that the simultaneous, siRNA-mediated down-regulation of UPAR and cathepsin B inhibits glioma cell migration and is accompanied by cytoskeletal condensation. We show that the dephosphorylation of cofilin is inhibited by the down-regulation of UPAR alone and, to a lesser extent, by the down-regulation of cathepsin B alone, and that the effect was much higher with the down-regulation of both molecules by pUC. Using FACS analysis and Western blotting for the $\alpha_{\mathrm{V}} \beta_{3}$ integrin heterodimer, we determined that down-regulating uPAR subsequently causes the down-regulation of the $\alpha_{V} \beta_{3}$ integrin heterodimer. As evidenced by Western blot analysis of ERK1/2, pERK1/2, p38MAPK, p-p38MAPK, AKT, pAKT and PI3-k, the MEK and PI3-k pathways are inhibited. From cytoskeleton studies, we observed that the down-regulation of uPAR caused cytoskeletal condensation and that the simultaneous downregulation of UPAR and cathepsin $B$ was even more effective at inducing cytoskeletal condensation than UPAR alone. Our results demonstrate the relevance of UPAR in cytoskeletal dynamics and the potential of UPAR and cathepsin B as targets in the treatment of malignant gliomas.
\end{abstract}

\section{Introduction}

Developing cancer cells acquire several capabilities including increased replicative potential, anchorage, independence

Correspondence to: Dr J.S. Rao, University of Illinois College of Medicine, One Illini Drive, Peoria, IL 61656, USA

E-mail: jsrao@uic.edu

Abbreviations: uPAR, urokinase-type plasminogen activator receptor; CMV, cytomegalovirus; $\mathrm{BGH}$, bovine growth hormone; PBS, phosphate-buffered saline; ECM, extracellular matrix; PAR-4, prostate apoptosis response-4; $\mathrm{ADF}$, actin depolymerizing factor

Key words: uPAR, cathepsin B, cofilin, actin, cytoskeleton, integrin from growth factors, evasion of apoptosis, angiogenesis, and invasion of surrounding tissue and metastasis (1). For a cell to divide, changes in cell architecture are required. These cellular changes are triggered by the cyclin B-CDK1/p34 complex, which phosphorylates proteins such as kinase-like motor proteins, which are required for mitotic spindle assembly, and caldesmon, an actin-binding protein $(2,3)$. Many researchers have indicated that the organization of the cytoskeleton is not restricted to mitosis alone and that cytoskeletal organization has been observed in the interphase.

A major component of the cytoskeleton is actin. Actin is one of the most abundant and highly conserved proteins among eukaryotes, the assembly/disassembly and organization of the actin cytoskeleton is regulated by actin-binding proteins including actin depolymerizing factor (ADF), also known as cofilin. Cofilins comprise a family of actin-binding proteins, which are ubiquitous among eukaryotes $(4,5)$. Mammals produce only three members of the Cofilin family: ADF, non-muscle cofilin, and muscle cofilin. All three mammalian cofilins contain a nuclear translocation sequence and can be targeted to the nucleus (6). Actin, on the other hand, has a nuclear export sequence rather than an import sequence suggesting that cofilin may be a nuclear chaperone for actin (7). The transformation of a normal cell into a cancer cell involves the enhancement of cell motility during metastasis and cell division. Both of these processes involve the condensation and reorganization of the actin cytoskeleton mediated by a decrease in adhesion-dependent growth. Cofilin is the final effector molecule that mediates the depolymerization of actin to be further processed for extension. Polymerization has been shown to be regulated by LIMK1/2 which, in turn, is regulated by the Ras-MEK pathway (8).

Disrupting the metastatic ability of cancer cells targeting the cytoskeleton architecture has been an attractive goal of cancer therapy (1). Our previous results have demonstrated the possible involvement of Ras-PI3k in the uPAR-mediated signaling cascade (9). We have shown that the absence of uPAR in human glioma cells leads to morphological changes associated with decreased spreading and a disorganized cytoskeleton resulting in altered cell morphology (10). Cathepsin B is known to be associated with uPAR and its integrin-mediated signaling ability (11). Hence, targeting uPAR and cathepsin B would mediate a signaling cascade, which should involve the rearrangement of the cytoskeleton. 
We have previously shown that the RNAi-mediated downregulation of $\mathrm{UPAR}$ and cathepsin $\mathrm{B}$ retards invasion and migration in SNB19 glioma cells (12). In the same study, we demonstrated that the down-regulation of UPAR and cathepsin B subsequently causes the dephosphorylation of ERK1/2 and a slight decrease in total ERK1/2 levels. Other groups have demonstrated that both migration and invasion by cells involves the de- and re-organization of the cytoskeleton (1). Thus, it is possible that targeting UPAR and its associated molecules, such as UPA or cathepsin B, will induce a change in cytoskeletal architecture and thereby retard invasion and migration. In this study, we have attempted to establish a link between integrinmediated UPAR signaling and the regulation of cofilin, which is involved in actin depolymerization.

\section{Materials and methods}

Plasmid construction. pcDNA 3 was used for the construction of a vector expressing siRNA for both cathepsin B and UPAR downstream of the cytomegalovirus (CMV) promoter. The uPAR sequence from +77 to +98 was used as the target sequence and, for convenience, a self-complimentary oligo was used. A uPAR sequence, 21 bases in length with a nine base loop region with BamHI sites incorporated at the ends (gatcctacagcagtggagagcgattatatataataatcgctctccactgctgtag), was used. The oligo was self-annealed in 6' SSC per standard protocol and ligated on to the BamHI site of a pcDNA-3 vector plasmid. Similarly, a cathepsin B complimentary sequence from +732 to +753 (tcgaggtggcctctatgaatcccaatatata attgggattcatagaggccacc) with $X h o I$ sites incorporated at the ends was ligated into the XhoI site of the vector containing the siRNA sequence for UPAR. This finally resulted in an siRNA expression plasmid for cathepsin B and UPAR designated as pUC. Single siRNA expression vectors for uPAR (puPAR) and cathepsin B (pCath B) were also constructed as described by our group (12). The orientation of either insert in the single or bicistronic was not relevant since the oligos were self-complimentary and had bilateral symmetry. BGH poly A terminator served as a stop signal for RNA synthesis for all three constructs.

Cell culture and transfection conditions. The SNB19 cell line, established from a human high-grade glioma, was used for this study. Cells were grown in Dulbecco's modified Eagle's medium/F12 media (1:1, v/v) supplemented with $10 \%$ fetal calf serum in a humidified atmosphere containing $5 \% \mathrm{CO}_{2}$ at $37^{\circ} \mathrm{C}$. SNB19 cells were transfected with empty vector (EV), scrambled vector (SV) puPAR, pCath B or pUC using lipofectamine as per the manufacturer's instructions (Life Technologies, Rockville, MD).

Western blot analysis. SNB19 cells were transfected with mock, empty vector, puPAR, pCath B or pUC and cultured for 48 h. Cells were then harvested, washed twice with cold PBS and lysed in buffer (150 mM NaCl, $50 \mathrm{mM}$ Tris- $\mathrm{HCl}, 2 \mathrm{mM}$ EDTA, $1 \%$ NP-40, pH 7.4) containing protease inhibitors. Equal amounts of protein $(30 \mu \mathrm{g} /$ lane $)$ from supernatants or cells were electrophoresed under non-reducing conditions on $10 \%$ acrylamide gels. After SDS-PAGE, proteins were transferred to a polyvinylidene difluoride membrane (Bio-Rad).
To block non-specific binding, the membrane was incubated for $2 \mathrm{~h}$ in PBS with $0.1 \%$ Tween-20 (T-PBS) containing 5\% non-fat skim milk. Subsequently, the membrane was incubated for $2 \mathrm{~h}$ with antibody against cathepsin B, uPAR, MAPK, phosMAPK, PI3k, PAR4, cofilin and phospho-cofilin in T-PBS $+5 \%$ non-fat milk. After washing in T-PBS, protein on the membrane was visualized using the $\mathrm{ECL}^{\mathrm{TM}}$ detection kit with a peroxidase-labeled anti-rabbit or anti-mouse antibody (Amersham Pharmacia Biotech, Amersham, UK) per manufacturer's instructions. For loading control, the membranes were stripped and probed with monoclonal antibodies for GAPDH as per standard protocol.

FACS analysis. SNB19 cells $\left(2 \times 10^{6}\right)$ were seeded on vitronectin-coated plates as described previously (9). Cells were transfected with EV/SV, puPAR, pCath B or pUC. Cells were trypsinized $72 \mathrm{~h}$ after transfection, washed with PBS, and resuspended at a concentration of $1 \times 10^{6}$ cells $/ \mathrm{ml}$ in PBS. Cells were then incubated with either control (mouse IgG) antibody (Santa Cruz, sc-2025) or LM609, an $\alpha_{\mathrm{V}} \beta_{3}$ integrin heterodimer-specific monoclonal antibody (Chemicon Int., Temecula, CA; 1:250 dilution), for $1 \mathrm{~h}$ on ice, pelleted, and washed three times with PBS to remove excess primary antibody. Cells were then resuspended in $1 \mathrm{ml}$ of PBS and incubated with biotinylated anti-mouse IgG (Vector Laboratories Inc., Burlingame, CA; 1:250 dilution) for $1 \mathrm{~h}$ on ice. After three more washes, streptavidin-fluorescein isothiocyanate conjugates (Life Technologies, Inc.; 1:150 dilution) were added, the cells were washed three times, and the cell pellet was resuspended in $2 \%$ paraformaldehyde and analyzed on a flow cytometer.

Visualization of actin cytoskeleton. SNB19 cells $\left(1 \times 10^{4}\right)$ were seeded on vitronectin-coated 8-well chamber slides, incubated for $24 \mathrm{~h}$ and transfected with EV/SV, puPAR, pCath B or pUC. Cells were washed with PBS, fixed with $3.7 \%$ formaldehyde, and permeabilized with $0.2 \%$ Triton X-100 for $5 \mathrm{~min}$. Then, F-actin was labeled by adding tetramethylrhodamine isothiocyanate-labeled phalloidin (200 ng/ml; Sigma) in PBS for $30 \mathrm{~min}$, and immunofluorescence was recorded photographically on a fluorescence microscope fitted with the appropriate filters. The condensation of the cytoskeleton was quantified as the percent of cells showing actin cytoskeleton condensation.

\section{Results}

Down-regulation of $u P A R$ and cathepsin B simultaneously cause the down-regulation of phospho AKT, phospho p38 (MAPK) and PI3-K. To determine the effect of RNAi-mediated down-regulation of uPAR and cathepsin B on SNB19 human glioma cells, the cells were transfected with mock, empty vector, puPAR, pCath B or pUC and cultured for $48 \mathrm{~h}$. At the end of incubation, cells were harvested, washed twice with cold PBS, lysed, and proteins were extracted as described earlier. From the Western blot analysis, it is clear that SNB19 cells transfected with puPAR showed a decrease in the expression of uPAR protein levels and transfection of SNB19 cells with pCath $B$ resulted in the down-regulation of cathepsin B protein levels as compared to the controls. Transfection of SNB19 


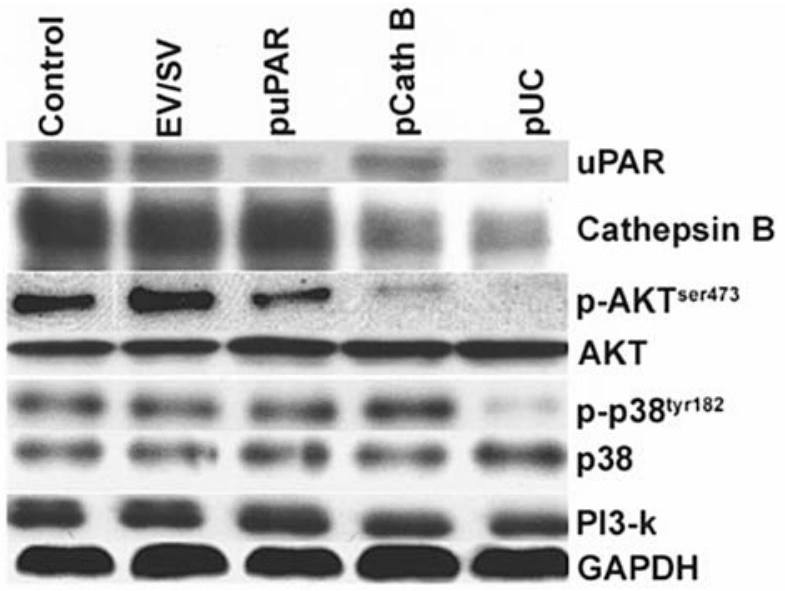

Figure 1. Western blot analysis of SNB19 cells transfected with mock, empty vector, puPAR, pCath B or pUC for cathepsin B, UPAR, MAPK, phospho-MAPK, AKT, p-AKT and PI3-K. Briefly, cells were harvested, washed twice with cold PBS, and lysed in buffer $(150 \mathrm{mM} \mathrm{NaCl}, 50 \mathrm{mM}$ Tris- $\mathrm{HCl}, 2 \mathrm{mM}$ EDTA, $1 \% \mathrm{NP}-40, \mathrm{pH}$ 7.4) containing protease inhibitors. Equal amounts of protein $(30 \mu \mathrm{g} / \mathrm{lane})$ from supernatants or cells were electrophoresed under non-reducing conditions on $10 \%$ acrylamide gels. After SDS-PAGE, proteins were transferred to a polyvinylidene difluoride membrane. To block non-specific binding, the membrane was incubated for $2 \mathrm{~h}$ in PBS with $0.1 \%$ Tween-20 (T-PBS) containing 5\% non-fat skim milk. Subsequently, the membrane was incubated for $2 \mathrm{~h}$ with antibody against cathepsin B, uPAR, p38, phospho-p38, AKT, pAKT and PI3-k in T-PBS $+5 \%$ non-fat milk, followed by appropriate HRP-conjugated secondary antibody. For loading control, the membranes were stripped and probed with monoclonal antibodies for GAPDH as per standard protocol.

cells with pUC resulted in the down-regulation of both uPAR and cathepsin B as compared to the controls. EV/SV-transfected SNB19 cells did not show any significant changes in uPAR and cathepsin B expression (Fig. 1). The protein levels of AKT and phospho-AKT ${ }^{\text {ser } 473}$ were also determined in mock-, empty vector-, puPAR-, pCath B- and pUC-transfected cells. AKT levels remained similar in mock-, empty vector-, puPAR-, pCath B- and pUC-transfected cells whereas phospho AKT ${ }^{\text {ser473 }}$ levels were reduced to almost undetectable in pUC-transfected cells. In puPAR-transfected cells, we observed a $~ 50 \%$ reduction in the levels of phospho-AKT ${ }^{\mathrm{ser} 473}$ as compared to the controls. The effect was more pronounced in pCath Btransfected cells with a $\sim 75 \%$ reduction in phospho-AKT ${ }^{\text {ser473 }}$ when compared to the controls. p38MAP kinase levels remained unchanged in mock-, empty vector-, puPAR-, pCath B- and pUC-transfected cells; whereas the levels of phospho-p38 $8^{\text {trl } 182}$ MAP kinase were reduced in pUC-transfected cells but remained unchanged in mock-, EV-, puPAR- and pCath B-transfected cells. The levels of PI3-k essentially remained unchanged in mock-, EV- and puPAR-transfected cells but were reduced by $\sim 20 \%$ in pCath B and $\sim 40 \%$ in pUC-transfected cells. GAPDH served as loading control.

The down-regulation of UPAR and cathepsin B is associated with a corresponding increase in PAR-4 levels. To determine the effect of RNAi-mediated down-regulation of UPAR and cathepsin B on SNB19 human glioma cells, the cells were transfected with mock, empty vector, puPAR, pCath B or pUC and cultured for $48 \mathrm{~h}$. At the end of incubation, the cells were harvested, washed twice with cold PBS, and lysed and proteins were extracted as described in Materials and methods.
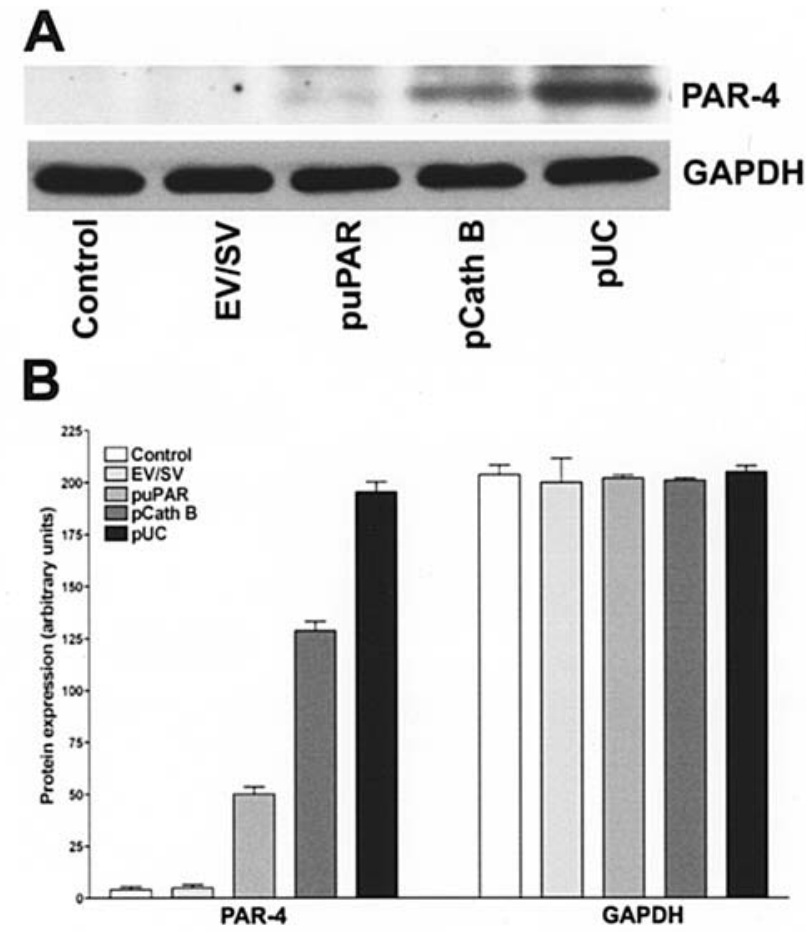

Figure 2. Western blot analysis of SNB19 cells transfected with mock, empty vector, puPAR, pCath B and pUC for PAR-4 and GAPDH. (A) Briefly, cells were harvested, washed twice with cold PBS, and lysed in buffer (150 mM NaCl, 50 mM Tris-HCl, 2 mM EDTA, 1\% NP-40, pH 7.4) containing protease inhibitors. Equal amounts of protein $(30 \mu \mathrm{g} / \mathrm{lane})$ from supernatants or cells were electrophoresed under non-reducing conditions on $10 \%$ acrylamide gels. After SDS-PAGE, proteins were transferred to a polyvinylidene difluoride membrane. To block non-specific binding, the membrane was incubated for $2 \mathrm{~h}$ in PBS with $0.1 \%$ Tween-20 (T-PBS) containing $5 \%$ non-fat skim milk. Subsequently, the membrane was incubated for $2 \mathrm{~h}$ with antibody against PAR-4 or GAPDH, in T-PBS $+5 \%$ non-fat milk, followed by appropriate HRP-conjugated secondary antibody. For loading control, the membranes were stripped and probed with monoclonal antibodies for GAPDH, as per standard protocol. (B) The Western blots were quantified and the expression levels of PAR-4, and GAPDH are represented as arbitrary units depending on the density of signal on the autoradiogram.

The down-regulation of uPAR alone did not induce any change in the levels of ERK1/2 or pERK1/2 as was shown previously (12), whereas the levels of PAR-4 did show a slight increase in expression with controls showing no detectable levels (Fig. 2A). In the case of cells transfected with pCath B, detectable levels of PAR-4 were observed with a corresponding decrease in ERK1/2 but no significant change in pERK1/2 levels (12). In pUC-transfected SNB19 cells, PAR-4 expression was observed and was $\sim 35 \%$ higher than in the pCath Btransfected cells (Fig. 2). GAPDH levels served as loading controls (Fig. 2).

$\alpha_{\bigvee} \beta_{3}$ integrin is down-regulated in correlation with the downregulation of $U P A R$ and cathepsin $B$ and is accompanied by the de-organization of actin cytoskeleton. To determine the effect of the down-regulation of UPAR and cathepsin B on the regulation of $\alpha_{V} \beta_{3}$ integrin, SNB19 cells $\left(2 \times 10^{6}\right)$ were seeded on vitronectin-coated plates as described previously (9). Fig. 3A is a graphical representation of the SNB19 cell population indicating the relative concentration of $\alpha_{V} \beta_{3}$ integrin in the SNB19 cell population. Isomatch IgG anti- 
A

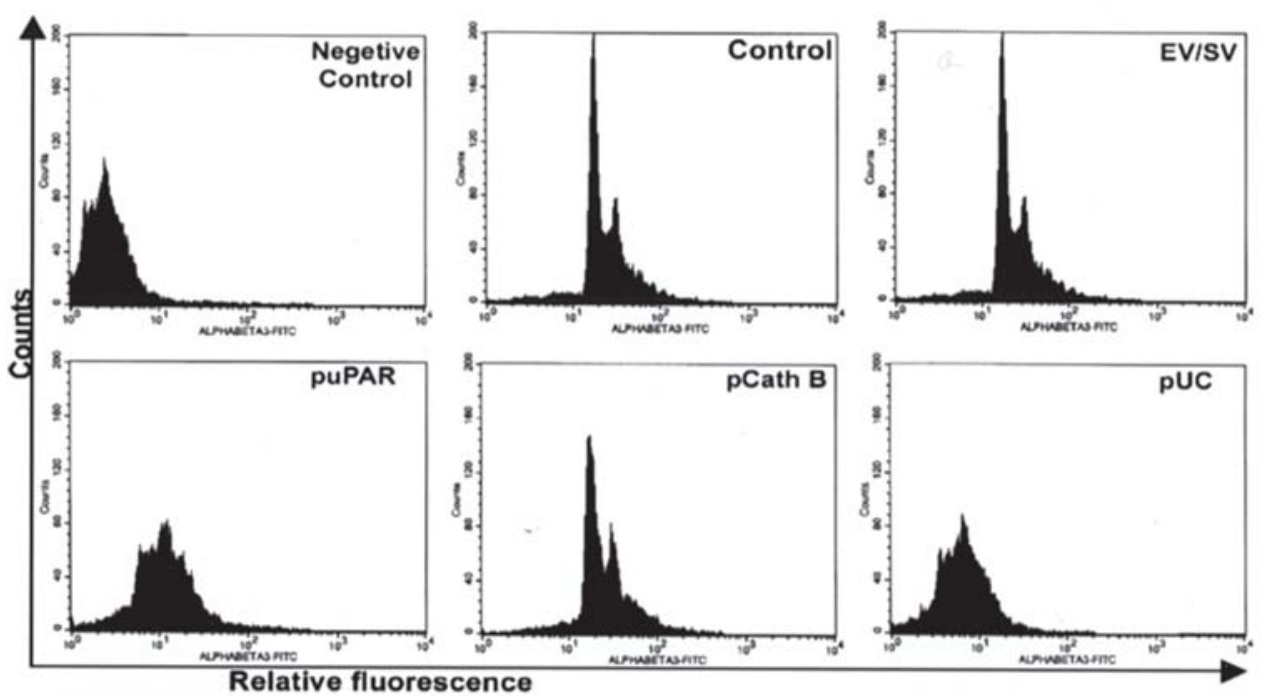

B

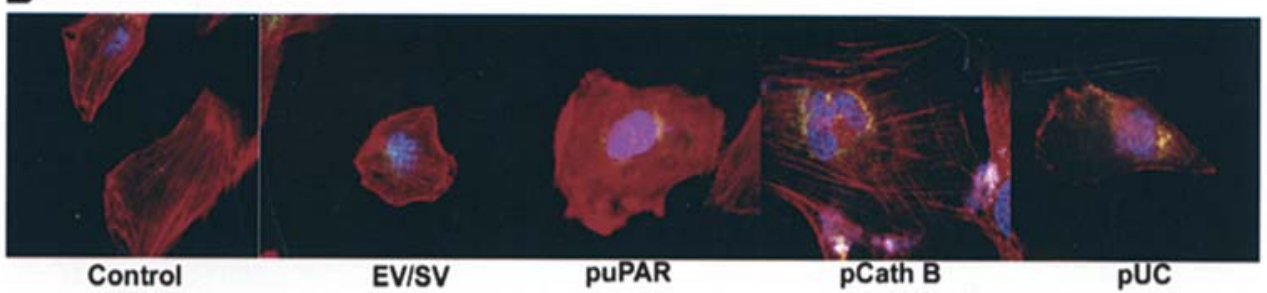

C
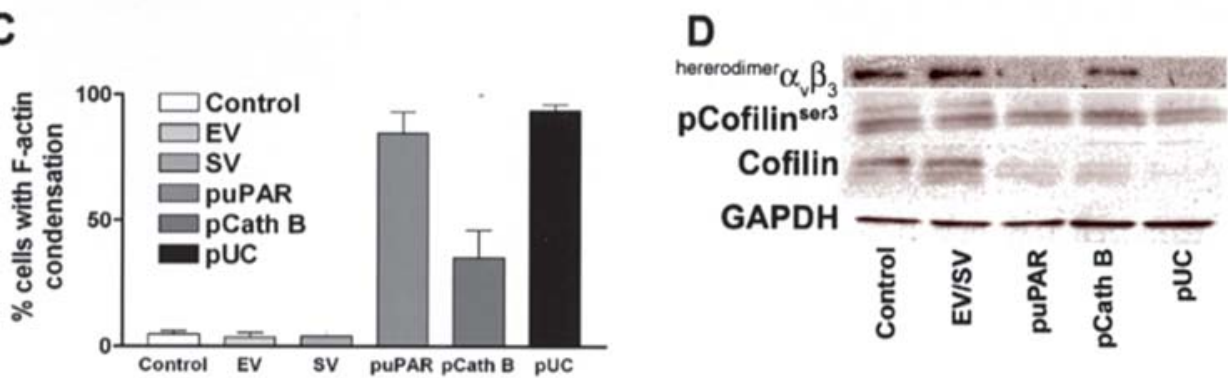

Figure 3. The simultaneous down-regulation of uPAR and cathepsin B induces the down-regulation of $\alpha_{v} \beta_{3}$ heterodimer, induces the condensation of F-actin and causes the phosphorylation of Cofilin. (A) SNB19 cells $\left(2 \times 10^{6}\right)$ were seeded on vitronectin-coated plates as described previously (9) and transfected with EV/SV, puPAR, pCath B or pUC. Cells were trypsinized $72 \mathrm{~h}$ after transfection, washed with PBS, and resuspended at a concentration of $1 \mathrm{x} 10^{6}$ cells/ml in PBS. Cells were then incubated with either control (mouse IgG) antibody (Santa Cruz, sc-2025) or LM609, an $\alpha_{v} \beta_{3}$ integrin heterodimer-specific monoclonal antibody for $1 \mathrm{~h}$ on ice, pelleted, and washed three times with PBS to remove excess primary antibody. Cells were then resuspended in $1 \mathrm{ml}$ of PBS and incubated with biotinylated anti-mouse IgG (Vector Laboratories Inc., Burlingame, CA; 1:250 dilution) for $1 \mathrm{~h}$ on ice. After three more washes, streptavidinfluorescein isothiocyanate conjugates (Life Technologies, Inc.; 1:150 dilution) were added, the cells were washed three times again, and the cell pellet was resuspended in $2 \%$ paraformaldehyde and analyzed on a flow cytometer. (B) F-actin condensation was visualized after staining with tetramethylrhodamine isothiocyanate-labeled phalloidin (200 ng/ml; Sigma) in PBS for $30 \mathrm{~min}$, and immunofluorescence was recorded on a fluorescence microscope fitted with the appropriate filters. (C) Briefly, SNB19 cells (1x104) were seeded on vitronectin-coated 8-well chamber slides, incubated for $24 \mathrm{~h}$ and transfected with EV/SV, puPAR, pCath B and pUC. Cells were washed with PBS, fixed with $3.7 \%$ formaldehyde, and permeabilized with $0.2 \%$ Triton X-100 for 5 min. Then, F-actin was labeled by adding tetramethylrhodamine isothiocyanate-labeled phalloidin ( $200 \mathrm{ng} / \mathrm{ml}$; Sigma) in PBS for 30 min, and immunofluorescence was recorded photographically on a fluorescence microscope fitted with the appropriate filters. The condensation of the cytoskeleton was quantified as the percent of cells showing actin cytoskeleton condensation. (D) Western blot analysis of the $\alpha_{\mathrm{v}} \beta_{3}$ integrin heterodimer, cofilin and phospho-cofilin was performed as described in Materials and methods. GAPDH served as loading control.

body was used for the negative control; untreated cells and EV/SV-treated cells were similar in their distribution of $\alpha_{V} \beta_{3}$ integrin. In contrast, the granularity of the cells was disrupted (y-axis) and a drop in the fluorescence was also observed (x-axis) in puPAR-treated cells. In pCath B-treated cells, the fluorescence remained essentially the same but there was a decrease in the granularity. Cells treated with pUC were similar to puPAR-treated cells. However, the degree of fluorescence was reduced much more in pUC-treated cells than in puPAR- treated cells. Similar to the levels of $\alpha_{V} \beta_{3}$ integrin heterodimer, phalloidin TRIT-C stained F-actin showed condensation in puPAR- and pUC-treated cells (Fig. 3B). Control and EV/SVtreated cells did not show significant morphological changes, whereas puPAR and pUC-treated cells showed condensation of F-actin with pUC-treated cells showing relatively lower concentrations of F-actin than the controls. In pCath B-treated cells, F-actin condensation was observed but was significantly less than that in pUC- and puPAR-treated cells. Quantative 


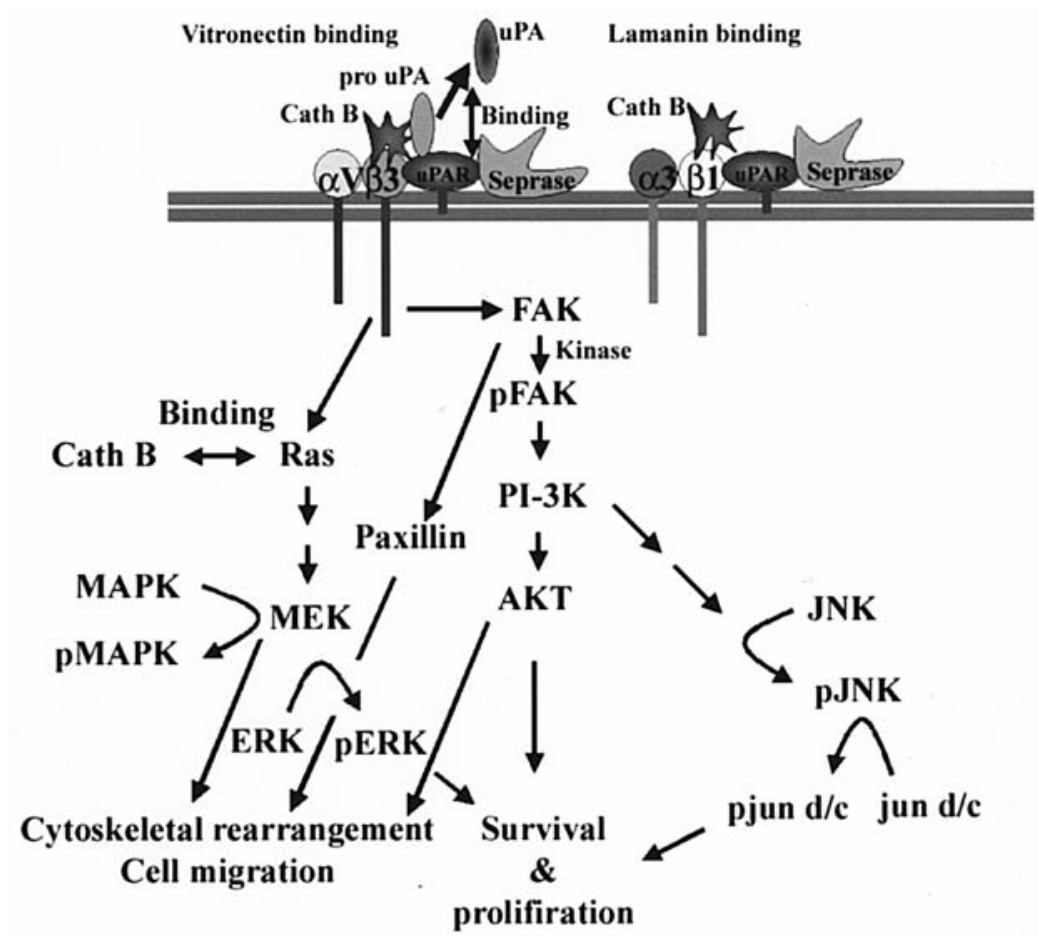

Figure 4. Schematic representation of the possible mechanisms involved in actin cytoskeleton regulation as initiated by uPAR and cathepsin B.

analysis of F-actin condensation was performed by screening 10 fields and represented as percent condensation (Fig. 3C). Control and EV/SV-treated cells did not show significant F-actin condensation whereas $80 \pm 5 \%$ of puPAR-treated cells showed F-actin condensation and $37 \pm 7 \%$ of pCath B-treated cells showed F-actin condensation. The most significant numbers were observed with pUC-treated cells, where $95 \pm 3 \%$ showed F-actin condensation.

Down-regulation of $U P A R$ and cathepsin $B$ mediates a decrease in active cofilin. To determine the effect of RNAi-mediated down-regulation of UPAR and cathepsin B on SNB19 human glioma cells, the cells were transfected with mock, empty vector, puPAR, pCath B or pUC and cultured for 48 h. Fig. 3D shows a reduction in the levels of active cofilin in puPAR-, pCath B- and pUC-treated cells. Of note, the levels of cofilin in pUC-transfected cells were almost undetectable. Cofilin expression in puPAR-treated cells was less than that in pCath B-treated cells. Similar levels of P-cofilin were observed in control, puPAR-, pCath B-, pUC-, and SV-treated cells (Fig. 3D). $\alpha_{V} \beta_{3}$ levels were significantly decreased in puPARand pUC-treated cells and the effect was less in pCath B-treated cells than in control and EV/SV-treated cells (Fig. 3D).

\section{Discussion}

For glioma cells to migrate, a constant change in the cellular architecture is required. Cell migration is a finely choreographed event, which involves several distinct steps. Extension of protrusion in response to migratory stimuli is coupled to actin polymerization. Actin filaments possess barbed and pointed ends, the barbed end being the end at which actin monomers are incorporated during polymerization. For actin polymerization to occur, uncapping of the barbed end (13), severing of F-actin by cofilin (14), and polymerization of actin by $\operatorname{Arp} 2 / 3$ (15) is required.

In our study, we have simultaneously down-regulated UPAR and cathepsin B and observed the destabilization and condensation of actin filaments. Both UPAR and cathepsin B are known to be associated in close proximity to $\alpha_{\mathrm{v}} \beta_{3}$ integrins (11). This close proximity to $\alpha_{V} \beta_{3}$ integrins has been implicated in their ability to initiate signaling events. We observed from our Western blot analysis of SNB19 cells transfected with puPAR, pCath B and pUC that the activation of AKT by phosphorylation is inhibited. This indicates that the signaling cascade mediated by $\alpha_{V} \beta_{3}$ integrins is interfered with at the PI3-k pathway (16).

In addition, we observed that the simultaneous downregulation of UPAR and cathepsin B causes the inhibition of activation of p38MAPK, which is known to be mediated by MEK (17). Our results indicate that the association of uPAR to $\alpha_{\mathrm{V}} \beta_{3}$ integrin not only activates the PI3-k-mediated activation of AKT but also simultaneously activates the MEK-mediated p38MAPK/ERK pathway. The simultaneous down-regulation of UPAR and cathepsin B also resulted in the down-regulation of total ERK1/2 with a corresponding increase in PAR-4 protein levels. Earlier reports have indicated that the phosphorylated form of ERK1/2 negatively regulates the PAR-4 expression and PAR-4 negatively regulates the total ERK1/2 expression (18). We have demonstrated that PAR-4 regulation is negatively controlled by uPAR and cathepsin B and can be used as an indicator of the phosphorylation state of ERK1/2. This also explains the decrease in total ERK1/2 levels that we observed previously (12).

From our FACS analysis studies, we observed that the cellular content of $\alpha_{V} \beta_{3}$ integrin was reduced much less in cells 
with cathepsin B down-regulation than in the control. In the case of uPAR-down-regulated cells, a decrease in the levels of $\alpha_{\mathrm{V}} \beta_{3}$ integrin was observed which was more pronounced in cells with simultaneous uPAR and cathepsin B downregulation.

Previous studies have suggested the importance of cofilin in actin dynamics (19) and that the dephosphorylation of cofilin is initiated through extracellular signaling mediated by integrins. Our results demonstrated that the simultaneous down-regulation of UPAR and cathepsin B not only hinders the activation of cofilin but also down-regulates levels of the $\alpha_{V} \beta_{3}$ integrin heterodimer as seen from FACS analysis and Western blotting.

Other researchers have demonstrated that EGF stimulation may indicate a more complex regulatory mechanism for cofilin dephosphorylation (20). In the present study, we have demonstrated that cofilin activation is inhibited by the downregulation of UPAR and cathepsin $\mathrm{B}$. This results in a signaling cascade to LIMK1/2, which is responsible for the phosphorylation of cofilin. The protein, 14-3-3६, is known to bind to inactive cofilin (i.e. phosphorylated cofilin) preventing its dephosphorylation and may be involved in regulating its phosphorylated state (21). AIP1 also binds to cofilin (22) and stimulates the depolymerization activity of cofilin. However, this may not be the case in UPAR-cathepsin B down-regulated cells since the levels of active cofilin were almost undetectable. It is more likely that the dephosphorylation of cofilin is prevented or inhibited by an associated molecule, such as 14-3-3 6 .

From our results, it is evident that the down-regulation of uPAR alone caused the decrease in $\alpha_{V} \beta_{3}$ integrin and active cofilin levels, indicating that UPAR alone is necessary for normal cellular migration. This conclusion can be substantiated from our previous results where we demonstrated that the antisense-mediated down-regulation of UPAR alone causes a decrease in invasion, migration, and reduction in the levels of $\alpha_{\mathrm{V}} \beta_{3}$ integrin (9). In contrast, the down-regulation of cathepsin $\mathrm{B}$ alone did not induce significant changes in the levels of $\alpha_{\mathrm{V}} \beta_{3}$ integrin whereas the levels of active cofilin were reduced. This indicates that the presence of cathepsin $\mathrm{B}$ with uPAR on the cell surface is important in maintaining a balance in the active and inactive state of cofilin (Fig. 4).

Earlier reports provide conclusive evidence that the activation of cofilin is mediated through Ras and requires the combined activities of Ras effectors, MEK and PI3-k (23). Given the role of cofilin for a functional actin cytoskeleton, the need for tight regulation of cofilin is evident. We have demonstrated, for the first time to our knowledge, that the regulation of cofilin is in part regulated by integrin-linked UPAR and is a part of the Ras and PI3-k pathway. In this study, we have demonstrated that the down-regulation of UPAR and cathepsin B either separately or together inhibits the dephosphorylation of cofilin. This might indicate that the uPAR-integrin linked MEK and PI3-k cascade is associated with cellular migration, which is mediated by cofilin.

We previously demonstrated the relevance of UPAR in the migration of glioma cells $(9,16)$ as well as the change in cytoskeletal organization and the involvement of MEK and PI3-k pathways in migration (16). Enough evidence exists to indicate that cofilin is regulated by the uPAR-integrin linked
MEK and PI3-k pathways. There is also mounting evidence to suggest that cofilin regulation is associated with EGF receptors and has been demonstrated in chemotaxis (24). Other studies have provided evidence that the localized actin polymerization is independent of PI3-kinase and rho protein activity and requires Arp2/3 complex and cofilin function. This suggests that multiple pathways connected to cell surface receptor proteins regulate cofilin. Other groups have also demonstrated that actin nucleation promoting factor (NPF) neural Wiskott-Aldrich syndrome protein (N-WASP) binds the SH2- plus SH3-domain containing adaptor protein, Nck, in control and VEGF-treated cells (25). In vivo results have demonstrated that imaging of GFP-labeled metastatic tumor cells reveals cell orientation towards blood vessels (26). A recent report has provided evidence for the existence of a novel regulator of cofilin-mediated actin reorganization chronophin and its involvement in cofilin regulation during cell division (27). We are now beginning to understand the processes involved in cytoskeletal reorganization and the involvement of cell surface receptors in cytoskeletal dynamics. In conclusion, this study reveals the importance of UPAR and cathepsin B in cell migration and as potential targets in the treatment of metastatic tumors.

\section{Acknowledgements}

We thank Shellee Abraham for preparing the manuscript and Diana Meister and Sushma Jasti for reviewing the manuscript. This research was supported by National Cancer Institute grants CA 75557, CA 92393, CA 95058, CA 116708 and N.I.N.D.S. NS47699 and Caterpillar, Inc., OSF Saint Francis, Inc., Peoria, IL (to J.S.R.).

\section{References}

1. Hannigan G, Troussard AA and Dedhar S: Integrin-linked kinase: a cancer therapeutic target unique among its ILK. Nat Rev Cancer 5: 51-63, 2005

2. Blangy A, Lane HA, d'Herin P, Harper M, Kress M and Nigg EA: Phosphorylation by $\mathrm{p} 34 \mathrm{cdc} 2$ regulates spindle association of human Eg5, a kinesin-related motor essential for bipolar spindle formation in vivo. Cell 83: 1159-1169, 1995.

3. Ubersax JA, Woodbury EL, Quang PN, Paraz M, Blethrow JD, Shah K, Shokat KM and Morgan DO: Targets of the cyclindependent kinase Cdk1. Nature 425: 859-864, 2003.

4. Lappalainen P, Kessels MM, Cope MJ and Drubin DG: The ADF homology (ADF-H) domain: a highly exploited actinbinding module. Mol Biol Cell 9: 1951-1959, 1998.

5. Bamburg JR: Proteins of the ADF/cofilin family: essential regulators of actin dynamics. Annu Rev Cell Dev Biol 15: 185-230, 1999.

6. Nishida E, Iida K, Yonezawa N, Koyasu S, Yahara I and Sakai H: Cofilin is a component of intranuclear and cytoplasmic actin rods induced in cultured cells. Proc Natl Acad Sci USA 84: 5262-5266, 1987.

7. Bamburg JR and Wiggan OP: ADF/cofilin and actin dynamics in disease. Trends Cell Biol 12: 598-605, 2002.

8. Pawlak G and Helfman DM: MEK mediates v-Src-induced disruption of the actin cytoskeleton via inactivation of the RhoROCK-LIM kinase pathway. J Biol Chem 277: 26927-26933, 2002.

9. Adachi Y, Lakka SS, Chandrasekar N, Yanamandra N, Gondi CS, Mohanam S, Dinh DH, Olivero WC, Gujrati M, Tamiya T, Ohmoto T, Kouraklis G, Aggarwal B and Rao JS: Downregulation of integrin alpha(v)beta(3) expression and integrinmediated signaling in glioma cells by adenovirus-mediated transfer of antisense urokinase-type plasminogen activator receptor (uPAR) and sense p16 genes. J Biol Chem 276: 47171-47177, 2001. 
10. Chintala SK, Mohanam S, Go Y, Venkaiah B, Sawaya R, Gokaslan ZL and Rao JS: Altered in vitro spreading and cytoskeletal organization in human glioma cells by down-regulation of urokinase receptor. Mol Carcinog 20: 355-365, 1997.

11. Rao JS: Molecular mechanisms of glioma invasiveness: the role of proteases. Nat Rev Cancer 3: 489-501, 2003.

12. Gondi CS, Lakka SS, Dinh DH, Olivero WC, Gujrati M and Rao JS: RNAi-mediated inhibition of cathepsin B and uPAR leads to decreased cell invasion, angiogenesis and tumor growth in gliomas. Oncogene 23: 8486-8496, 2004.

13. Hartwig JH, Bokoch GM, Carpenter CL, Janmey PA, Taylor LA, Toker A and Stossel TP: Thrombin receptor ligation and activated Rac uncap actin filament barbed ends through phosphoinositide synthesis in permeabilized human platelets. Cell 82: 643-653, 1995.

14. Chan AY, Bailly M, Zebda N, Segall JE and Condeelis JS: Role of cofilin in epidermal growth factor-stimulated actin polymerization and lamellipod protrusion. J Cell Biol 148: 531-542, 2000.

15. Pollard TD, Blanchoin L and Mullins RD: Molecular mechanisms controlling actin filament dynamics in non-muscle cells. Annu Rev Biophys Biomol Struct 29: 545-576, 2000.

16. Chandrasekar N, Mohanam S, Gujrati M, Olivero WC, Dinh DH and Rao JS: Down-regulation of UPA inhibits migration and PI3k/Akt signaling in glioblastoma cells. Oncogene 22: 392-400, 2003.

17. Gellert GC, Goldfarb RH and Kitson RP: Physical association of uPAR with the alphaV integrin on the surface of human NK cells. Biochem Biophys Res Commun 315: 1025-1032, 2004.

18. Qiu SG, Krishnan S, El Guendy N and Rangnekar VM: Negative regulation of Par-4 by oncogenic Ras is essential for cellular transformation. Oncogene 18: 7115-7123, 1999.
19. Falet H, Chang G, Brohard-Bohn B, Rendu F and Hartwig JH: Integrin \{alpha\}IIb $\{$ beta\} 3 signals lead to cofilin to accelerate platelet actin dynamics. Am J Physiol Cell Physiol 289: C819$825,2005$.

20. Mouneimne G, Soon L, Des Marais V, Sidani M, Song X, Yip SC, Ghosh M, Eddy R, Backer JM and Condeelis J: Phospholipase $\mathrm{C}$ and cofilin are required for carcinoma cell directionality in response to EGF stimulation. J Cell Biol 166: 697-708, 2004.

21. Gohla A and Bokoch GM: 14-3-3 regulates actin dynamics by stabilizing phosphorylated cofilin. Curr Biol 12: 1704-1710, 2002.

22. Ono S: Regulation of actin filament dynamics by actin depolymerizing factor/cofilin and actin-interacting protein 1: new blades for twisted filaments. Biochemistry 42: 13363-13370, 2003.

23. Nebl G, Fischer S, Penzel R and Samstag Y: Dephosphorylation of cofilin is regulated through Ras and requires the combined activities of the Ras-effectors MEK and PI3K. Cell Signal 16: 235-243, 2004.

24. Kempiak SJ, Yip SC, Backer JM and Segall JE: Local signaling by the EGF receptor. J Cell Biol 162: 781-787, 2003.

25. Gong C, Stoletov KV and Terman BI: VEGF treatment induces signaling pathways that regulate both actin polymerization and depolymerization. Angiogenesis 7: 313-322, 2004.

26. Condeelis JS, Wyckoff JB, Bailly M, Pestell R, Lawrence D, Backer J and Segall JE: Lamellipodia in invasion. Semin Cancer Biol 11: 119-128, 2001

27. Gohla A, Birkenfeld J and Bokoch GM: Chronophin, a novel HAD-type serine protein phosphatase, regulates cofilin-dependent actin dynamics. Nat Cell Biol 7: 21-29, 2005. 